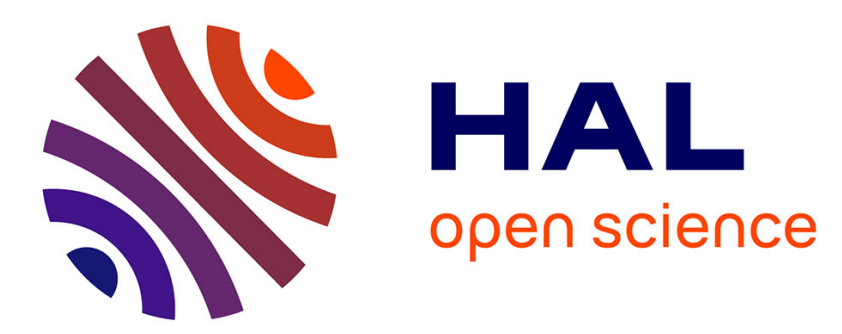

\title{
Symmetry-Driven Formation of Chiral Boroxine-Based Organometallic Oligomers on Ag(001)
}

Amelia Domínguez-Celorrio, Manuel Vilas-Varela, Diego Peña, Véronique Langlais, David Serrate

\section{To cite this version:}

Amelia Domínguez-Celorrio, Manuel Vilas-Varela, Diego Peña, Véronique Langlais, David Serrate. Symmetry-Driven Formation of Chiral Boroxine-Based Organometallic Oligomers on $\mathrm{Ag}(001)$. Journal of Physical Chemistry C, 2021, 125 (3), pp.2015 - 2021. 10.1021/acs.jpcc.0c08039 . hal-03396644

\section{HAL Id: hal-03396644 https://hal.science/hal-03396644}

Submitted on 27 Oct 2021

HAL is a multi-disciplinary open access archive for the deposit and dissemination of scientific research documents, whether they are published or not. The documents may come from teaching and research institutions in France or abroad, or from public or private research centers.
L'archive ouverte pluridisciplinaire HAL, est destinée au dépôt et à la diffusion de documents scientifiques de niveau recherche, publiés ou non, émanant des établissements d'enseignement et de recherche français ou étrangers, des laboratoires publics ou privés. 


\section{Symmetry driven formation of chiral boroxine-based organometallic oligomers on $\operatorname{Ag}(001)$}

Amelia Domínguez-Celorrio, $₫$ Manuel Vilas-Varela,§, Diego Peña,ई Véronique Langlais, $₫$

David Serrate. $\stackrel{\perp}{=}, *$

- Instituto de Nanociencia y Materiales de Aragón, CSIC-Universidad de Zaragoza, 50009

Zaragoza, Spain.

Centre d'Elaboration de Materiaux et d'Etudes Structurales, CNRS, 31055, Toulouse, France.

$\S$ Centro Singular de Investigación en Química Biolóxica e Materiais Moleculares (CiQUS), and Departamento de Química Orgánica, Universidade de Santiago de Compostela, 15782, Santiago de Compostela, Spain.

$\stackrel{\perp}{\perp}$ Departamento de Física de la Materia Condensada, Universidad de Zaragoza, 50009, Zaragoza, Spain.

$\dagger$ Laboratorio de Microscopias Avanzadas, Universidad de Zaragoza, 50018 Zaragoza, Spain.

\section{AUTHOR INFORMATION}

\section{Corresponding Author}

*E-mail: serrate@unizar.es (D.S.). 


\begin{abstract}
Two dimensional molecular assemblies on surfaces have opened a way to design and control chirality, featuring promising electronic and chemical properties that depend on the local handedness of the layer. Yet, the mechanisms leading to spontaneous chiral resolution are not fully understood at every reaction stage. Here, starting from achiral 10-bromoanthracene-9boronic acid as molecular precursor, we demonstrate enantiomeric induction in the products during the stage of covalent bonding, stemming from the competing point symmetries of the $\operatorname{Ag}(001)$ supporting surface and the reaction products. Upon dehalogenation and dehydration of precursors, hexagonal boroxine rings linked by organometallic anthracene dimers form, which undergo a strong interaction with four-fold symmetric substrate. The pro-chiral structural character of the resulting oligomer and its impact over the spatial distribution of the electronic molecular states are revealed by high resolution scanning tunneling microscopy and spectroscopy.
\end{abstract}




\section{INTRODUCTION}

Chiral crystallization of a racemic or achiral set of precursors is a striking phenomenon. Still, nature tends to depart spontaneously from random distributions of enantiomers. ${ }^{1,2} \mathrm{~A}$ necessary condition for chirality is the breaking of inversion symmetry, which can be achieved by carrying out the synthesis atop a surface. For instance, the surface of water droplets is thought to polymerize $2 \mathrm{D}$ crystallites of homochiral oligopeptides, a key ingredient of the prebiotic soup. ${ }^{3}$ The process of biomineralization, which often leads to chiral macrostructures, is explained in terms of asymmetric biomolecules interacting with the step edges of inorganic crystalline surfaces. ${ }^{4}$ Another advantage of using surfaces is the confinement of reactants to a $2 \mathrm{D}$ space, which lowers their degrees of freedom and allows minute energy differences between enantiomorphic geometries to induce spontaneous chiral resolution ${ }^{5-7}$ and chiral amplification. ${ }^{8}$ However, the vast majority of experimental studies about homochiral segregation on surfaces at the single molecule level is restricted to molecular self-assemblies where the building units are not covalently linked to each other. ${ }^{9-12}$ At the level of chemical bonding, understanding the role of a surface in chiral resolution requires the use of a well-controlled $2 \mathrm{D}$ reaction environment. For this purpose, the use of on-surface synthesis $(\mathrm{OSS})^{13}$ techniques is a very convenient strategy.

OSS is a powerful synthetic approach in vacuum where the supporting substrate catalyzes several steps and at the same time is used to steer the reaction towards low dimensional products confined to the surface plane. Molecular precursors with specific chemical functionality diffuse throughout the surface to form designated covalent molecular nanostructures. In this process, the 
constrains imposed by the molecule-surface and molecule-molecule interactions play a critical role. In the last decade OSS has shown its potential to produce organic macromolecules and graphene nanoribbons ${ }^{14,15}$ with atomically precise control over both the internal structure ${ }^{16,17}$ and the edges. ${ }^{18-20}$ Chiral precursors can naturally form planar prochiral (formally achiral molecules that become chiral by the desymmetrization introduced by the adsorption surface) molecular structures via OSS. ${ }^{20-22}$ Another possibility is that a heterochiral adsorption footprint ${ }^{6}$ breaks the inversion symmetry of achiral reactants, giving rise to purely electronic chirality in certain molecular orbitals ${ }^{7}$ or even to prochiral macromolecules via selective bond activation. ${ }^{23}$

Here we report the synthesis of a structurally prochiral organometallic framework (MOF) starting from achiral precursors, where the prochirality is induced by the different point symmetry of the substrate lattice and the polymer itself. Individual 10-bromoanthracene-9boronic acid (BABA) molecules were sublimated onto a clean $\mathrm{Ag}(001)$ surface kept at room temperature. Simultaneous debromination and dehydration of the $\mathrm{B}(\mathrm{OH})_{2}$ moieties $^{24,25}$ results in a polymeric network consisting in boroxine hexagons $\left(\mathrm{B}_{3} \mathrm{O}_{3}\right)$ interconnected by $\mathrm{C}-\mathrm{Ag}-\mathrm{C}$ organometallic (OM) bonds ${ }^{26}$ (see Fig. 1). The chirality appears as a consequence of the competition between the threefold symmetry of the $\mathrm{B}_{3} \mathrm{O}_{3}$ rings and the interaction of the $\mathrm{Ag}$ atoms with the four-fold symmetric substrate. This competition invariably leads to enantiomeric building units that undergo chiral recognition at the OM bonds. The proposed mechanism broadens the pool of chemical pathways to obtain chiral frameworks, and could be applied to the bottom-up synthesis of 2D chiral polymers with responsive behavior to chirality, such as aminoacid sensors, filters, or seeds to transfer the chirality into the third dimension for asymmetric catalysis. 


\section{EXPERIMENTAL METHODS}

Experiments were performed with an ultrahigh vacuum low temperature scanning tunneling microscope, the Joule-Thomson scanning tunneling microscopy (JT-STM) system (SPECS), with a pressure better than $1 \times 10^{-10}$ mbar. The current $\left(I_{t}\right)$ is measured in the tip, which is grounded, and we use sample bias $\left(V_{s}\right)$ convention. The atomically clean $\operatorname{Ag}(001)$ surface was

prepared by cycles of ionized argon sputtering at $1 \mathrm{kV}, 3 \times 10^{-6}$ mbar and subsequent annealing at $450^{\circ} \mathrm{C}$. The BABA molecules were sublimated at a temperature of $200{ }^{\circ} \mathrm{C}$ from a homemade resistive evaporator onto $\mathrm{Ag}(001)$ surface held at room temperature, regulating the deposition flux to $0.1-0.4 \mathrm{ML} / \mathrm{min}$ by means of a quartz microbalance. After deposition, the samples were transferred to a SPECS JT-STM operating at $4.2 \mathrm{~K}$. Differential conductance $d I / d V$ data were acquired using a lock-in amplifier at $913 \mathrm{~Hz}$ and modulation voltage of $8.8 \mathrm{mV}$, in constant height mode for the maps and constant current mode for point spectra. All images were analyzed using WSxM. ${ }^{27}$ The X-ray photoelectron spectra (XPS) of the precursor powder were measured on a XPS Spectrometer Kratos AXIS with an monochromatic incident radiation from a Al Ka source (Supplemental Figure S1).

\section{RESULTS AND DISCUSSION}

BABA molecules were synthesized following ref. ${ }^{28}$ and then sublimated onto a $\mathrm{Ag}(001)$ surface held at room temperature. Fig. 1a shows a STM image of a representative sample with two distinguishable phases. The chemical bifunctionality of BABA precursor including a bromine and a boronic acid moiety per molecule, opens the door to two different chemical reactions: Ullmann coupling and dehydration of boronic acids. Correspondingly, we find on one 
hand organometallic dimers (OD phase) that self-assemble into crystalline islands, and on the other hand, nested organometallic structures of linked boroxines with different lengths (MOF phase). The presence of these different phases suggests that there are two coexisting reaction paths (see Fig. 1b). Path A yields the formation of the organo-silver dimer, which is a wellknown intermediate of the Ullmann coupling on Ag surfaces, ${ }^{26,29}$ and typically precedes the covalent $\mathrm{C}-\mathrm{C}$ bonding after the reductive elimination step. In the OD phase the boronic acid is cleaved. Path B takes place when three BABA precursors or organo-silver dimers retaining the boronic acid group undergo a dehydration reaction, forming a 2D structure of boroxine rings ${ }^{30,31}$ linked by organometallic dimers. Deposition onto samples held at low temperatures could shed further insight into the reaction order of dehydration and debromination. As illustrated by Fig. 1a, in the MOF phase we have found oligomers of up to 14 boroxine units linked by organometallic dimers.

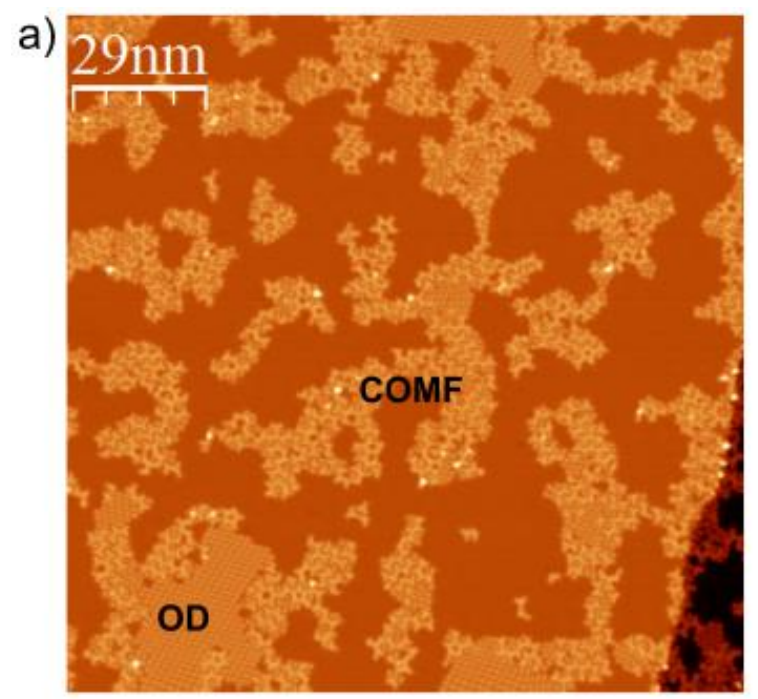

b)

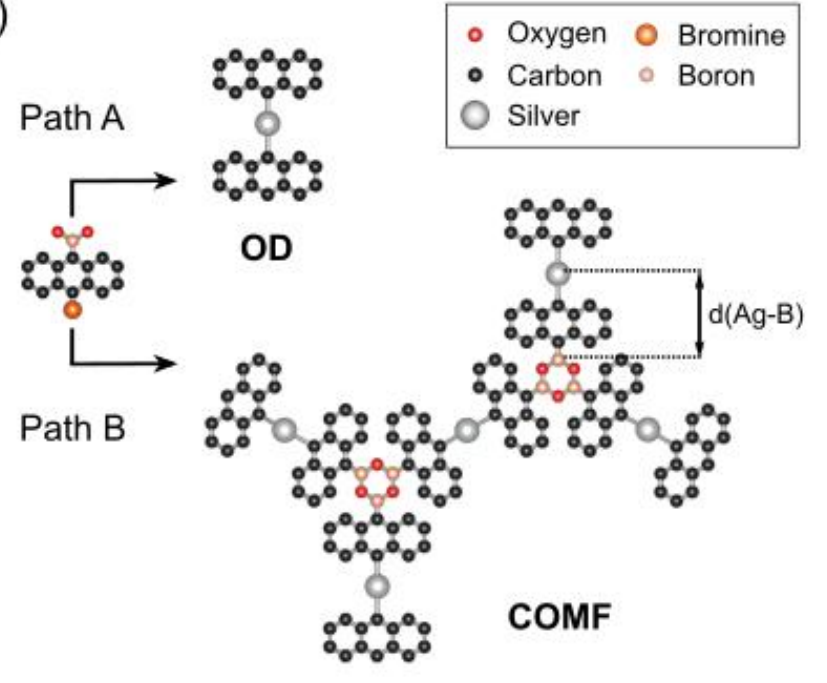

Figure 1. (a) Constant current STM image ( $\left.V_{s}=0.5 \mathrm{~V} ; I_{t}=0.05 \mathrm{nA}\right)$ of a representative BABA sample on $\operatorname{Ag}(001)$ showing two different phases: organometallic dimers phase (OD) and organometallic linked boroxines (MOF). (b) Schematic reaction pathways of the products 
obtained after BABA deposition on $\operatorname{Ag}(001)$ : organo-silver dimers (Path $\mathrm{A}$ ) and covalent organometallic framework of linked boroxines (Path B). d(Ag-B) points the distance between the metallic center of an organometallic dimer and the corresponding boron. The hydrogens are removed from the chemical structures for simplicity.

The absence of $\mathrm{B}_{3} \mathrm{O}_{3}$ rings when $\mathrm{BABA}$ are deposited on $\mathrm{Au}(111)$ (see Supplementary Note) ensures that the condensation into boroxines observed on $\operatorname{Ag}(001)$ does not take place in the crucible or during sublimation, as occurs with smaller boronic acids. ${ }^{32}$ In order to check whether the $\mathrm{B}-\mathrm{C}$ bonds in the pristine BABA powder are broken while heating up the crucible, we performed X-ray photoemission spectroscopy. We find no changes in either the binding energy of the B1s edge or the relative boron/carbon ratio (see Supplementary Fig. S1) after repeated sublimations of the powder. Hence, we conclude that most of the BABA precursors reach the $\operatorname{Ag}(001)$ surface intact. Under these conditions, we could expect that all precursors dehydrate on surface to form a boroxine, but instead we observe anthryl units bonded to boroxines $(38.2 \pm 1 \%$ as derived from statistical analysis of 6100 anthracenes) and no intact acid groups at all, which points towards a strong tendency to the $\mathrm{C}-\mathrm{B}$ cleavage of the $\mathrm{B}(\mathrm{OH})_{2}$ groups on coinage metal surfaces (see data on $\operatorname{Ag}(111)$ and $\mathrm{Au}(111)$ in Supplementary Note). This finding could be applied to implement Suzuki cross-coupling ${ }^{33}$ in OSS using similar precursors to produce graphene nanoribbons. In our case, the smooth anthracene edges at the position previously occupied by boron atoms (Fig. 2 and Supplemental Fig. S3), suggest that the C bonds are passivated by hydrogen after cleavage (for example coming from the OH-groups). For BABA on $\operatorname{Ag}(001)$, no single anthracene with intact boronic acid was found on the surface, suggesting that the formation of MOF strongly depends on the availability of pristine precursors to produce the 
dehydration of boronic acids before bonding to a silver adatom or losing the acid. On the other hand, $94 \pm 0.1 \%$ of the boroxine terminal anthracenes are part of an organo-silver dimer, evidencing that the condensation of boronic acids into a boroxine does not affect the reactivity of brominated carbons.

The dehydration of aryl boronic acid derivatives with halogen groups has also been observed on $\operatorname{Ag}(111) \cdot{ }^{32,34}$ However, in the case of $\operatorname{Ag}(001)$ the organo-silver dimers obtained at room temperature do not transform into C-C bonds, as is characteristic of the Ullmann coupling. Subsequent post-annealing does not help to initiate this second step because desorption takes place at $300^{\circ} \mathrm{C}$, before any substantial change of the MOF is observed (see Supplemental Figure S4). The high stability of the C-Ag bonds in both phases suggests that the steric repulsion between the hydrogens in vicinal rings of our planarly adsorbed precursors impedes the C-C bonding to form bianthracene moieties.
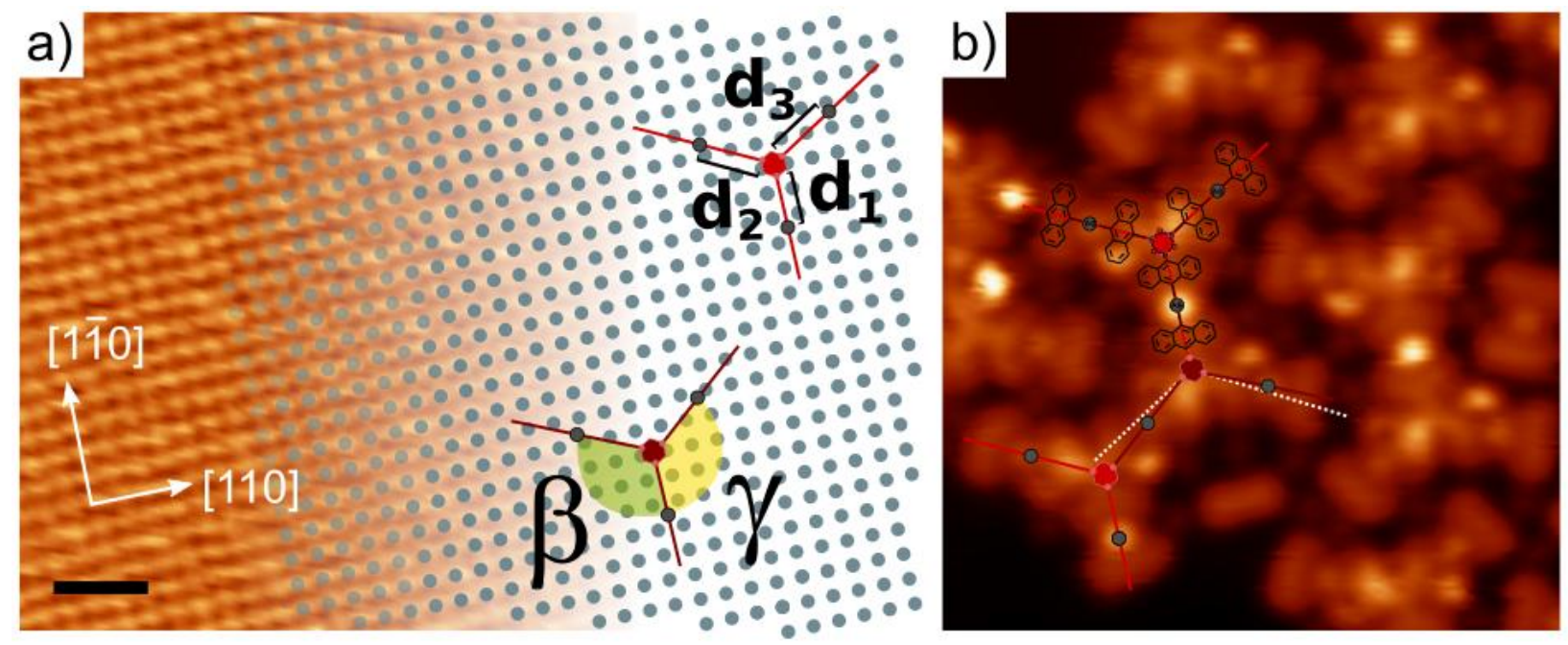

Figure 2. (a) STM topography of atomically resolved $\mathrm{Ag}(001)$ surface $(\mathrm{Vs}=20 \mathrm{mV}$; It $=50 \mathrm{nA})$ with the scheme of prochiral boroxines units of Models 1 and 2 (see Table 1) on $\operatorname{Ag}(001)$. The distances between the boron and the linked Ag atom (grey circles) are defined by $\mathrm{d}_{1}$ (along [110] 
direction), $d_{2}$ and $d_{3} . \beta$ and $\gamma$ are the experimental angles between [110] direction and the other two organometallic axes. (b) Constant height STM image of an island with hexagonal truncated phase $\left(V_{s}=5 \mathrm{mV}\right)$ showing chiral building blocks in (a) superimposed. White dotted lines represent the boroxine axis every $120^{\circ}$. Scale bar: $1 \mathrm{~nm}$.

The MOF geometry is strongly influenced by the interaction of the substrate with the remaining Ag atoms between anthracenes. Fig. 2a shows a high-resolution image of a short MOF with three linked boroxines. The Ag atoms appear as round protrusions surrounded by two elongated anthryl units, which are arranged around the boroxine centers and exhibit bonding axes at approximately $120^{\circ}$. The seven $\mathrm{Ag}$ atoms contained in the structure do not have the same appearance. The two Ag atoms bonded to anthracenes along the [1-10] direction of the substrate lattice (Fig. 2b) are clearly brighter than the others. This is an electronic fingerprint for different adsorption sites. Because of the higher symmetry of the dimers along $\{110\}$ directions, we assume that they correspond to the energetically most favorable adsorption geometry. Considering that the $\mathrm{sp}^{2}$ boron orbitals are oriented with an angle of $120^{\circ}$ between each other, the bonding axes of the two other branches of the boroxine should exhibit a threefold symmetric arrangement. However, this is prohibited by the fourfold symmetry of the substrate they are strongly interacting with. The arrangement of the other two branches will have to minimize at the same time the adsorption energy of the remaining Ag adatoms, and the energy penalty due to the distortion of the C-B-O bonding angles away from $120^{\circ}$. The independent optimization of both terms is not allowed, forcing in this way a compromise for the adsorption site of the $\mathrm{Ag}$ atoms between the fourfold and the threefold symmetries. Thus, attending to the sign of the angle formed by the actual B-Ag segment and the axes at $120^{\circ}$ from the [110] direction (corresponding to the ideal hexagonal structure, white dotted lines in Fig. 2b), each building block composed by 
one $\mathrm{B}_{3} \mathrm{O}_{3}$ ring and its three organo-silver dimers can be classified into a chiral rotational sense. For example, the two buildings blocks found in Fig 2 exhibit $R$-handedness.

The minimal structural model that allows us to fit our high-resolution images consists in three lines pinned at the boron atoms of a perfect hexagonal boroxine. The Ag atoms are placed at the middle point of the lines. The only free parameters are defined in Figure 2a: (i) the relative angles between the dimer axis along $\{110\}$ and the axes of the other two branches $(\beta, \gamma)$; (ii) the B-Ag distance along the other two branches $\left(\mathrm{d}_{2}\right.$ and $\left.\mathrm{d}_{3}\right)$. The length of organo-silver dimers is kept constant to a value of 0.76(9) $\mathrm{nm}$ as extracted from the analysis of individual dimers in the OD phase (Supplemental Figure S3b). The size of the boroxine hexagon is set to the value of one benzene ring. Finally, the inter-boron distance along $\{110\}$ directions $\left(2 \mathrm{~d}_{1}\right)$ is also fixed in our model to $1.32(1) \mathrm{nm}$. This value was calculated as the measured inter-boroxine distance along $\{110\}$ directions minus the length of the boroxine hexagon. Note that this model is equivalent to fixing the B-C bond length and C-B-O angle along $\{110\}$ directions to their experimental values and letting free the other two. This model is fitted to STM constant-height current images of different sample areas where creep and thermal drift are relaxed below a negligible level of less than $1 \mathrm{pm}$ during the scan of a MOF unit as the one shown in Fig. 2a.

In Fig. 2a we superimpose the fit of our structural model to one building block. The comparison clearly shows that $\beta \neq \gamma \neq 120^{\circ}$. Since high symmetry positions cannot be occupied by the $\mathrm{Ag}$ atoms in branches 2 and 3 , several structural models could exist, each one corresponding to a metastable adsorption geometry (see for instance the Ag sites in the models sketched in Figure 2a). We have identified three of them (as well as their corresponding enantiomers, see an example at Supplementary Fig. S5) by analyzing a total of 86 boroxine building blocks in high 
resolution images of the same quality as Fig. 2a. The structural parameters for these models are given in Table 1. The building blocks are always prochiral, with deviations ranging $0.5(5)^{\circ}<\mid \beta$ $120 \mid<8.5(5)^{\circ}$. In general, $\mathrm{d}_{1}, \mathrm{~d}_{2}$ and $\mathrm{d}_{3}$ are different. Because our model assumption that the size of the organo-silver dimer yields a perfect fit of the images (see sketch in Figure 3), the different Ag-B distances imply that C-B bond length depends on the linking direction.

\begin{tabular}{lccccc}
\hline Trimer & Handedness & $\boldsymbol{\beta}\left({ }^{\mathbf{o}}\right)$ & $\boldsymbol{\gamma}\left({ }^{\mathbf{0}}\right)$ & $\mathbf{d}_{\mathbf{2}} / \mathbf{d}_{\mathbf{1}}$ & $\mathbf{d}_{\mathbf{3}} / \mathbf{d}_{\mathbf{1}}$ \\
\hline Model 1 & $R$ & 117 & 120.5 & $1.21(3)$ & $1.22(5)$ \\
Model 1 & $S$ & 120.5 & 117 & $1.22(5)$ & $1.21(3)$ \\
Model 2 & $R$ & 115 & 128.5 & $1.20(6)$ & $1.05(1)$ \\
Model 2 & $S$ & 128.5 & 115 & $1.05(1)$ & $1.20(6)$ \\
Model 3 & $R$ & 115 & 122.5 & $1.23(2)$ & $0.98(1)$ \\
Model 3 & $S$ & 122.5 & 115 & $0.98(1)$ & $1.23(2)$
\end{tabular}

Table 1. Structural parameters of prochiral building blocks of MOF. All the models and the parameters have been extracted measuring on high resolution STM images. Angles are determined with an error of $0.5^{\circ}$. Model 1 and 2 are sketched in Figure $2 \mathrm{a}$ (red and wine respectively), model 3 is sketched in Figure 3 (pink).

As shown in Figure 3, MOFs self-assemble into homochiral domains of the order of $10 \mathrm{~nm}$. The MOF phase island in this example is in contact with the OD phase, which is a general trend in 
our samples (see Fig. 1a). Here, we identify up to three levels of chiral recognition. First, we note that the $\mathrm{C}-\mathrm{Ag}-\mathrm{C}$ bonding direction in the branches with low adsorption symmetry is univocally determined by $\beta$ and $\gamma$ (see Fig. 2a). Henceforth, these angles fix the growth directions in which a MOF can bind to another building block via OM bonds. The link only occurs when $\beta$ or $\gamma$ in the reacting building blocks coincide, which permits a straight organometallic bond. This precludes reactions between building blocks of different chirality (see the large angle difference between enantiomers in Table 1) along directions away from $\{110\}$. This enantioselectivity is therefore imposed by geometric constrains. Second, in the case of CAg-C bonds along $\{110\}$ directions, we also find asymmetric synthesis in all MOFs. In this direction, however, there is not any geometric burden to link building blocks with different chirality, because anthracenes and $\mathrm{Ag}$ atoms have a unique adsorption geometry that is totally independent from $\beta$ and $\gamma$. This provides strong evidence that the enantioselectivity in the MOF growth is not only due to geometric constrains, but rather has an underlying electronic component, say, a formation energy that depends on the chiral character of the molecular orbitals and favors the OM bonding between boroxine building blocks with the same chiral character. Although $a b$ initio calculations would be necessary to understand the interaction mechanism, in our work this electronic effect is supported by the energy resolved spectroscopy discussed hereinafter. In addition, a similar effect has been previously reported for weak intermolecular electronic interactions driving homochiral self-assembly of $\mathrm{Cu}$-phtalocyanines. ${ }^{7}$ 


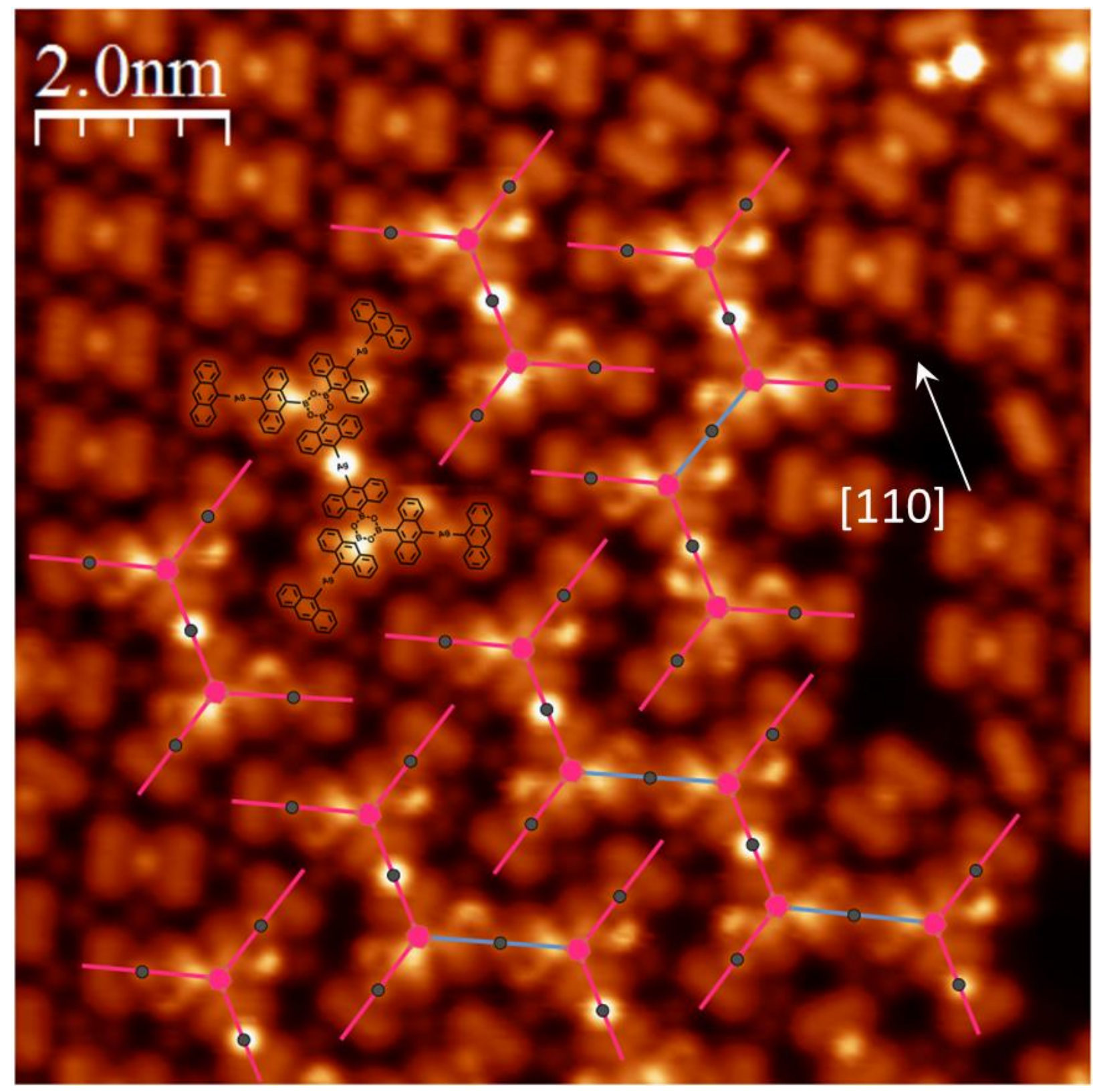

Figure 3. Constant height STM image of a self-assembled MOF island $\left(V_{s}=5 \mathrm{mV}\right)$. The chemical structure and the schematic $(R)$-Model 3 (see Table 1) are overlaid on prochiral $(R)$ organometallic polymer fragments. Blue lines represent dimers linking boroxines in directions different from $\{110\}$. In these cases, since $2 \mathrm{~d}_{2}$ does not match with full precision the particular inter-boroxine distance, the Ag-B distance adapts with some tensile strain of about $5 \%$. 
Finally, we observe domains where all individual MOFs have the same handedness (Figure 3a). This spontaneous segregation in homochiral domains is attributed to halogen-mediated selfassembly, previously reported for other molecules on noble metal substrates. ${ }^{8,35}$ In our case, $\mathrm{Br}$ atoms are clearly imaged as round protrusions between MOFs, occupying equivalent positions relative to the building blocks in a regular pattern. This suggests a strong interaction between $\mathrm{H}$ terminated carbons and the free halogen atoms left over the surface after cleaving the $\mathrm{Br}-\mathrm{C}$ bonds. This interaction, of electrostatic origin, may play a relevant role during the formation building blocks, favoring the availability of neighboring units with the same chirality.
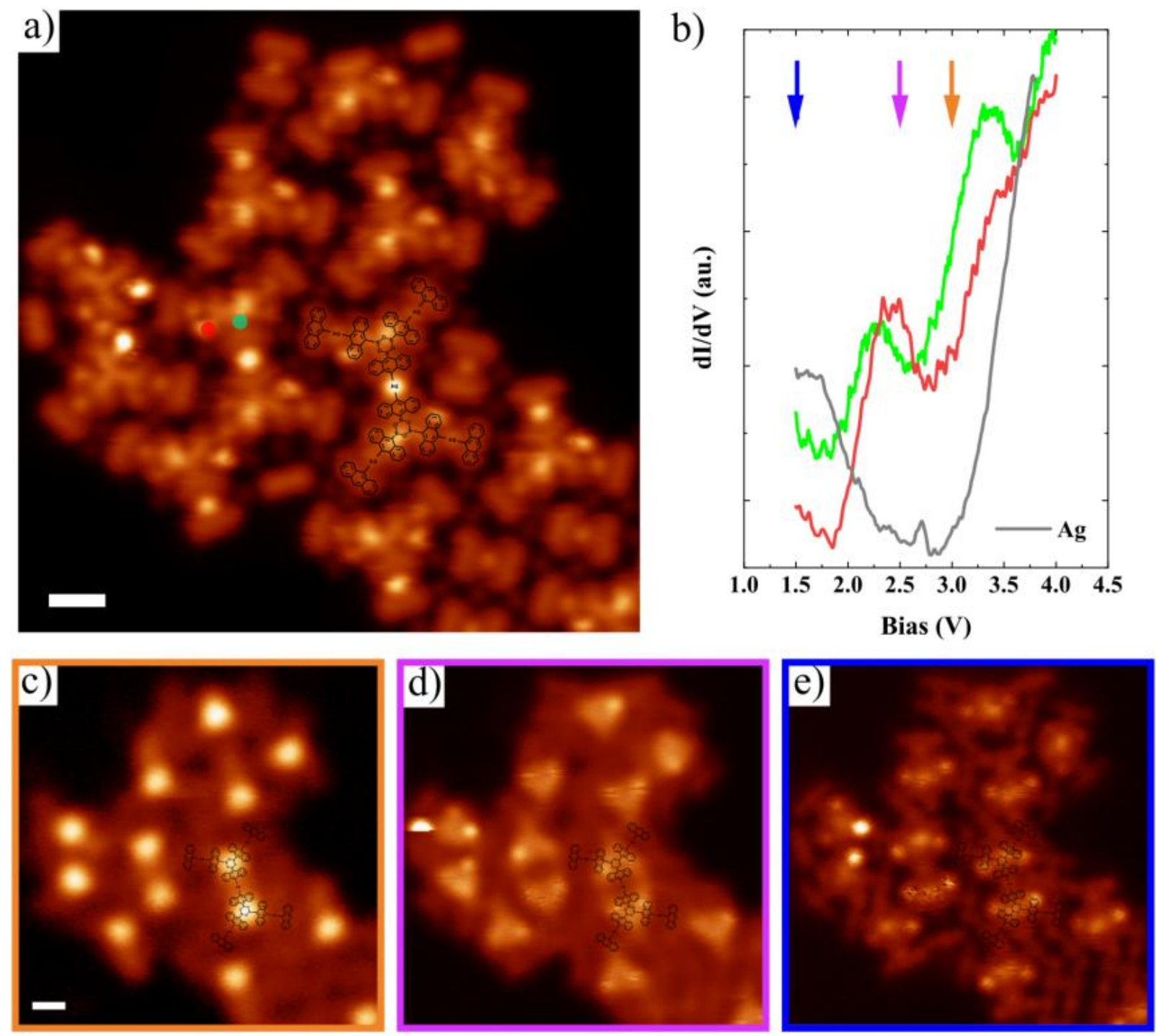
Figure 4. (a) Constant height STM image of MOF with (R)-Model 1 and (R)-Model 2 as building blocks $\left(V_{s}=5 \mathrm{mV}\right)$. (b) Constant current $\mathrm{d} I / \mathrm{d} V$ spectra on phenyl/boroxine sites (red/green curves), acquired in the organometallic oligomer at the positions marked by filled circles in (a) $\left(V_{\text {mod }}=9 \mathrm{mV}\right.$, initial regulation set point $\left.V_{s}=500 \mathrm{mV}, I_{t}=25 \mathrm{pA}\right)$. (c-e) Constant height $\mathrm{d} I / \mathrm{d} V$ maps of a selected region in (a), scanning parameters: $V_{s}=3 \mathrm{~V}(\mathrm{c}), 2.5 \mathrm{~V}(\mathrm{~d})$ and $V$ $=1.5 \mathrm{~V}(\mathrm{e})$. The chemical structure of a MOF with two boroxines is overlaid on the STM images as a guide the eye. Scale bars: $1 \mathrm{~nm}$.

In order to probe the electronic structure of chiral MOFs and its likely participation in the enantioselective OM bonding, we performed differential conductance $(\mathrm{d} I / \mathrm{d} V)$ tunneling spectroscopy. Point spectra acquired over selected locations (shown in Figure 4a-b) exhibited features at $3.3 \mathrm{eV}$ and $2.2 \mathrm{eV}$ associated with boroxines. Another molecular orbital at $2.45 \mathrm{eV}$ is visible at the position of outer phenyl rings in chiral branches. Constant height $\mathrm{d} I / \mathrm{d} V$ images at these energies allowed us to map the spatial distribution of the observed molecular states. As shown in Figure 4c, the boroxine state at 3.3 V displays a marked circular shape. This supports our assumption of undistorted hexagonal $\mathrm{B}_{3} \mathrm{O}_{3}$ rings in the structural model. The boroxine states show some weaker intensity distributed across the OM dimers along the [110] axis. This delocalization is not observed across the OM dimers lying along low symmetry directions. On the other hand, the orbital at $2.45 \mathrm{~V}$ (Figure $4 \mathrm{~d}$ ) has a triangular shape with sides coincident with the anthracenes bound to a boroxine ring. The corners of these triangles are approximately located at the terminal hydrogens of the anthracenes, and exhibit different intensities, which could be attributed to some tilting with respect to the surface plane.

Additional orbitals pop up in the constant height maps at $1.5 \mathrm{eV}$ (Figure 4e), which are not visible in point spectra due to the intrinsic $\operatorname{Ag}(001)$ contribution at this energy range (grey curve 
in Figure $4 \mathrm{~b}$ ). These orbitals can be described as three lobe structures around the boroxines, being reminiscent of the $2.45 \mathrm{eV}$ molecular state. Two lobes lie just outside the phenyl rings, and another one on top of a B-C bond, all three belonging to the chiral branches of the building block. In this state, the previously observed chirality in the structural analysis becomes evident in the electronic structure too. The $1.5 \mathrm{~V}$ state overlaps in space with the boroxine state, which in turn has been found to connect boroxines along $\{110\}$ directions. This indirect overlap of the chiral orbitals through the boroxine orbitals (which does not discard the existence of additional chiral selective chemical interactions which are not visualized in our spectroscopy data), provides a rationale for the enantiospecific links along these directions in the absence of geometric constrains. The $1.5 \mathrm{~V}$ map displays a very faint intensity over the $\mathrm{Br}$ atoms as well.

\section{CONCLUSIONS}

In conclusion, the competition between the internal symmetries of an on-surface synthesized macromolecule and the supporting substrate provides a mechanism to confer chirality on the otherwise achiral product. Chiral recognition during the MOF growth is ascribed to geometric constrains and to the sensitivity of orbital overlap to the chiral character of the basic building blocks. We also highlight the role of halogen bonds in the homochiral segregation of selfassembled MOF islands. This work shows a strategy to create chiral organometallic networks from achiral precursors directly on the surface. We also evidence how Ullmann coupling intermediates can adopt chiral configurations depending on their interaction strength with surface provided that there are different point symmetries. 


\section{SUPPORTING INFORMATION DESCRIPTION}

The following files are available free of charge as Supporting information. -babaAg001_SI_v3.0 (PDF file): Peripherial data on $\mathrm{Au}(111)$ and $\mathrm{Ag}(111)$ and comments including XPS precursor powder characterization and image sequence illustrating the effect of annealing no $\operatorname{Ag}(001)$

\section{AUTHOR INFORMATION}

The authors declare no competing financial interests.

\section{ACKNOWLEDGMENT}

Financial support was provided by the European Union through Interreg-POCTEFA (Grant TNSI/EFA194/16) and Horizon 2020 (Grant Agreement No. 863098 (“SPRING”)) programs, including EFRD funds, as well as by the Spanish Plan Nacional de I+D+i (MAT2016-78293-C6 and PID2019-107338RB-C6). We also acknowledge funding from Gobierno de Aragón (grupo de referencia E13_20R "NANOMIDAS") and Xunta de Galicia (Centro singular de investigación de Galicia, accreditation 2016-2019, ED431G/09).

\section{REFERENCES}

(1) Avalos, M.; Babiano, R.; Cintas, P.; Jimenez, J. L.; Palacios, J. C. Symmetry Breaking by Spontaneous Crystallization - Is It the Most Plausible Source of Terrestrial Handedness We Have Long Been Looking for? - A Reappraisal. Orig Life Evol Biosph 2004, 34 (4), 391405. https://doi.org/10.1023/B:ORIG.0000029886.32034.f3.

(2) Green, M. M.; Park, J.-W.; Sato, T.; Teramoto, A.; Lifson, S.; Selinger, R. L. B.; Selinger, J. V. The Macromolecular Route to Chiral Amplification. Angew. Chem. Int. Ed. 1999, 17.

(3) Weissbuch, I.; Bolbach, G.; Leiserowitz, L.; Lahav, M. Chiral Amplification of Oligopeptides via Polymerization in Two-Dimensional Crystallites on Water. Orig Life Evol Biosph 2004, 34 (1/2), 79-92. https://doi.org/10.1023/B:ORIG.0000009830.40297.fe. 
(4) Orme, C. A.; Noy, A.; Wierzbicki, A.; McBride, M. T.; Grantham, M.; Teng, H. H.; Dove, P. M.; DeYoreo, J. J. Formation of Chiral Morphologies through Selective Binding of Amino Acids to Calcite Surface Steps. Nature 2001, 411 (6839), 775-779. https://doi.org/10.1038/35081034.

(5) Karageorgaki, C.; Mutombo, P.; Jelinek, P.; Ernst, K.-H. Chiral Surface from Achiral Ingredients: Modification of $\mathrm{Cu}(110)$ with Phthalic Acid. J. Phys. Chem. C 2019, 123 (14), 9121-9127. https://doi.org/10.1021/acs.jpcc.9b00637.

(6) Forster, M.; Dyer, M. S.; Persson, M.; Raval, R. Tailoring Homochirality at Surfaces: Going Beyond Molecular Handedness. J. Am. Chem. Soc. 2011, 133 (40), 15992-16000. https://doi.org/10.1021/ja202986s.

(7) Mugarza, A.; Lorente, N.; Ordejón, P.; Krull, C.; Stepanow, S.; Bocquet, M.-L.; Fraxedas, J.; Ceballos, G.; Gambardella, P. Orbital Specific Chirality and Homochiral Self-Assembly of Achiral Molecules Induced by Charge Transfer and Spontaneous Symmetry Breaking. Phys. Rev. Lett. 2010, 105 (11), 115702. https://doi.org/10.1103/PhysRevLett.105.115702.

(8) Fasel, R.; Parschau, M.; Ernst, K.-H. Amplification of Chirality in Two-Dimensional Enantiomorphous Lattices. Nature 2006, $439 \quad$ (7075), 449-452. https://doi.org/10.1038/nature04419.

(9) Zaera, F. Chirality in Adsorption on Solid Surfaces. Chem. Soc. Rev. 2017, 46 (23), 7374 7398. https://doi.org/10.1039/C7CS00367F.

(10) Raval, R. Molecular Assembly at Surfaces: Progress and Challenges. Faraday Discuss. 2017, 204, 9-33. https://doi.org/10.1039/C7FD90072D.

(11) Stetsovych, O.; Švec, M.; Vacek, J.; Chocholoušová, J. V.; Jančařík, A.; Rybáček, J.; Kosmider, K.; Stará, I. G.; Jelínek, P.; Starý, I. From Helical to Planar Chirality by OnSurface Chemistry. Nature Chemistry 2017, 9 (3), 213-218. https://doi.org/10.1038/nchem.2662.

(11) Xiang, F.; Lu, Y.; Wang, Z.; Ju, H.; Filippo, G. D.; Li, C.; Liu, X.; Leng, X.; Zhu, J.; Wang, L.; et al. On-Surface Synthesis of Chiral $\pi$-Conjugate Porphyrin Tapes by SubstrateRegulated Dehydrogenative Coupling. The Journal of Physical Chemistry C 2019. https://doi.org/10.1021/acs.jpcc.9b06025.

(13) Clair, S.; de Oteyza, D. G. Controlling a Chemical Coupling Reaction on a Surface: Tools and Strategies for On-Surface Synthesis. Chem. Rev. 2019, 119 (7), 4717-4776. https://doi.org/10.1021/acs.chemrev.8b00601.

(13) Cai, J.; Ruffieux, P.; Jaafar, R.; Bieri, M.; Braun, T.; Blankenburg, S.; Muoth, M.; Seitsonen, A. P.; Saleh, M.; Feng, X.; et al. Atomically Precise Bottom-up Fabrication of Graphene Nanoribbons. Nature 2010, $466 \quad$ (7305), 470-473. https://doi.org/10.1038/nature09211.

(15) Grill, L. Large Molecules on Surfaces: Deposition and Intramolecular STM Manipulation by Directional Forces. Journal of Physics: Condensed Matter 2010, 22 (8), 084023. https://doi.org/10.1088/0953-8984/22/8/084023.

(16) Hieulle, J.; Carbonell-Sanromà, E.; Vilas-Varela, M.; Garcia-Lekue, A.; Guitián, E.; Peña, D.; Pascual, J. I. On-Surface Route for Producing Planar Nanographenes with Azulene Moieties. Nano Letters 2018, $18 \quad$ (1), 418-423. https://doi.org/10.1021/acs.nanolett.7b04309.

(16) Mishra, S.; Beyer, D.; Eimre, K.; Liu, J.; Berger, R.; Gröning, O.; Pignedoli, C. A.; Müllen, K.; Fasel, R.; Feng, X.; et al. Synthesis and Characterization of $\pi$-Extended Triangulene. $J$. Am. Chem. Soc. 2019, 141 (27), 10621-10625. https://doi.org/10.1021/jacs.9b05319. 
(17) Ruffieux, P.; Wang, S.; Yang, B.; Sánchez-Sánchez, C.; Liu, J.; Dienel, T.; Talirz, L.; Shinde, P.; Pignedoli, C. A.; Passerone, D. et al. On-Surface Synthesis of Graphene Nanoribbons with Zigzag Edge Topology. Nature 2016, 531 (7595), 489-492. https://doi.org/10.1038/nature17151.

(19) Chen, Y.-C.; Cao, T.; Chen, C.; Pedramrazi, Z.; Haberer, D.; de Oteyza, D. G.; Fischer, F. R.; Louie, S. G.; Crommie, M. F. Molecular Bandgap Engineering of Bottom-up Synthesized Graphene Nanoribbon Heterojunctions. Nature Nanotechnology 2015, 10 (2), 156-160. https://doi.org/10.1038/nnano.2014.307.

(19) Wang, X.-Y.; Urgel, J. I.; Barin, G. B.; Eimre, K.; Di Giovannantonio, M.; Milani, A.; Tommasini, M.; Pignedoli, C. A.; Ruffieux, P.; Feng, X.; et al. Bottom-Up Synthesis of Heteroatom-Doped Chiral Graphene Nanoribbons. Journal of the American Chemical Society 2018. https://doi.org/10.1021/jacs.8b06210.

(20) de Oteyza, D. G.; García-Lekue, A.; Vilas-Varela, M.; Merino-Díez, N.; CarbonellSanromà, E.; Corso, M.; Vasseur, G.; Rogero, C.; Guitián, E.; Pascual, J. I.; et al. SubstrateIndependent Growth of Atomically Precise Chiral Graphene Nanoribbons. ACS Nano 2016, 10 (9), 9000-9008. https://doi.org/10.1021/acsnano.6b05269.

(22) Merino-Díez, N.; Mohammed, M. S. G.; Castro-Esteban, J.; Colazzo, L.; BerdoncesLayunta, A.; Lawrence, J.; Pascual, J. I.; de Oteyza, D. G.; Peña, D. Transferring Axial Molecular Chirality through a Sequence of On-Surface Reactions. Chem. Sci. 2020, 10.1039.D0SC01653E. https://doi.org/10.1039/D0SC01653E.

(23) Sánchez-Sánchez, C.; Dienel, T.; Deniz, O.; Ruffieux, P.; Berger, R.; Feng, X.; Müllen, K.; Fasel, R. Purely Armchair or Partially Chiral: Noncontact Atomic Force Microscopy Characterization of Dibromo-Bianthryl-Based Graphene Nanoribbons Grown on $\mathrm{Cu}(111)$. ACS Nano 2016, 10 (8), 8006-8011. https://doi.org/10.1021/acsnano.6b04025.

(24) Schlögl, S.; Sirtl, T.; Eichhorn, J.; Heckl, W. M.; Lackinger, M. Synthesis of TwoDimensional Phenylene-Boroxine Networks through in Vacuo Condensation and onSurface Radical Addition. Chemical Communications 2011, 47 (45), 12355. https://doi.org/10.1039/c1cc13896k.

(25) Clair, S.; Abel, M.; Porte, L. Growth of Boronic Acid Based Two-Dimensional Covalent Networks on a Metal Surface under Ultrahigh Vacuum. Chem. Commun. 2014, 50 (68), 9627-9635. https://doi.org/10.1039/C4CC02678K.

(26) Simonov, K. A.; Generalov, A. V.; Vinogradov, A. S.; Svirskiy, G. I.; Cafolla, A. A.; McGuinness, C.; Taketsugu, T.; Lyalin, A.; Mårtensson, N.; Preobrajenski, A. B. Synthesis of Armchair Graphene Nanoribbons from the 10,10'-Dibromo-9,9'-Bianthracene Molecules on $\operatorname{Ag}(111)$ : The Role of Organometallic Intermediates. Sci Rep 2018, 8 (1), 3506. https://doi.org/10.1038/s41598-018-21704-3.

(27) Horcas, I.; Fernández, R.; Gómez-Rodríguez, J. M.; Colchero, J.; Gómez-Herrero, J.; Baro, A. M. WSXM: A Software for Scanning Probe Microscopy and a Tool for Nanotechnology. Review of Scientific Instruments 2007, 78 (1), 013705. https://doi.org/10.1063/1.2432410.

(28) Filthaus, M.; Oppel, I. M.; Bettinger, H. F. Supramolecular Structures and Spontaneous Resolution: The Case of Ortho-Substituted Phenylboronic Acids. Org. Biomol. Chem. 2008, 6 (7), 1201. https://doi.org/10.1039/b719282g.

(29) Eichhorn, J.; Strunskus, T.; Rastgoo-Lahrood, A.; Samanta, D.; Schmittel, M.; Lackinger, M. On-Surface Ullmann Polymerization via Intermediate Organometallic Networks on 
Ag(111). Chem. Commun. 2014, $50 \quad$ (57), https://doi.org/10.1039/C4CC02757D.

(30) Faury, T.; Dumur, F.; Clair, S.; Abel, M.; Porte, L.; Gigmes, D. Side Functionalization of Diboronic Acid Precursors for COF. UHV and TGA Opposite Reactivities. CrystEngComm 2013, 15 (11), 2067. https://doi.org/10.1039/c3ce26494g.

(30) Ourdjini, O.; Pawlak, R.; Abel, M.; Clair, S.; Chen, L.; Bergeon, N.; Sassi, M.; Oison, V.; Debierre, J.-M.; Coratger, R.; et al. Substrate-Mediated Ordering and Defect Analysis of a Surface Covalent Organic Framework. Phys. Rev. B 2011, 84 (12), 125421. https://doi.org/10.1103/PhysRevB.84.125421.

(32) Schlögl, S.; Sirtl, T.; Eichhorn, J.; Heckl, W. M.; Lackinger, M. Synthesis of TwoDimensional Phenylene-Boroxine Networks through in Vacuo Condensation and onSurface Radical Addition. Chemical Communications 2011, 47 (45), 12355. https://doi.org/10.1039/c1cc13896k.

(33) Miyaura, N.; Suzuki, A. Stereoselective Synthesis of Arylated (E)-Alkenes by the Reaction of Alk-1-Enylboranes with Aryl Halides in the Presence of Palladium Catalyst. J. Chem. Soc., Chem. Commun. 1979, No. 19, 866. https://doi.org/10.1039/c39790000866.

(34) Faury, T.; Clair, S.; Abel, M.; Dumur, F.; Gigmes, D.; Porte, L. Sequential Linking To Control Growth of a Surface Covalent Organic Framework. The Journal of Physical Chemistry C 2012, 116 (7), 4819-4823. https://doi.org/10.1021/jp300417g.

(34) Lu, J.; Bao, D.-L.; Dong, H.; Qian, K.; Zhang, S.; Liu, J.; Zhang, Y.; Lin, X.; Du, S.-X.; $\mathrm{Hu}, \mathrm{W}$; et al. Construction of Two-Dimensional Chiral Networks through Atomic Bromine on Surfaces. J. Phys. Chem. Lett. 2017, 8 (2), 326-331. https://doi.org/10.1021/acs.jpclett.6b02680. 
TOC GRAPHICS

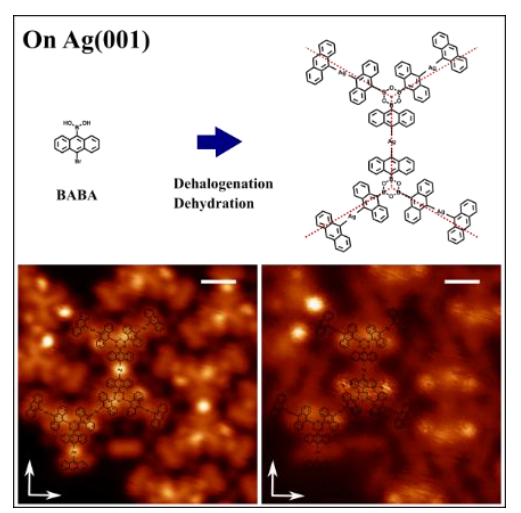

